\title{
Vitamine $D$, de la vitamine à l'hormone
}

Au-delà de ses propriétés dans le métabolisme du calcium, la vitamine $D$ figure parmi les agents immunomodulateurs et inducteurs de la différenciation des cellules sanguines.

Livia Miravet

Directeur de recherches à l'Inserm $\left(U_{I} 8\right)$.

\section{François Wilhelm}

Chef de clinique-Assistant, service de rhumatologie de l'hôpital Lariboisière.

\section{REFERENCES}

I. Mac I aughlin JA, Anderson RR, Holick MP Spectral character of sunlight modulates photosynthesis of previtamin $\mathrm{D}$, and its photoisomers in human skin. Science 1982; 216 : 1001-3.

2. Miravet L, Rambaud JC, Louis C, Hioco D. Absorption de la vitamine $\mathrm{D}_{3}{ }^{3} \mathrm{H}$ dans les ostéomalacies nutritionnelles. Sem Hop Paris $1969 ; 45: 531-538$.

3. Carre M, Miravet L, Hioco D. Absorption de la vitamine $\mathrm{D}_{3}$ étudiée à l'aide d'une solution micellaire de la vitamine $D_{3}{ }^{3} \mathrm{H}$ dans l'anse intestinale isolée de rat in situ. Comptes Rendus de la Société de Biologie (Paris). 1972; $166: 843-8$.

4. Fuicushima M, Nishii Y, Suzuki M, Suda T. Comparative studies on the 25-hydroxylations of cholecalciferol and i $\alpha$ hydroxycholecalciferol in perfused rat liver. Biochem 7 1978; 170 : 495-9.

\section{ADRESSE}

L. Miravet, F. Wilhelm : Inserm U 18 , hôpital Lariboisière, 6, rue Guy-Patin, 75010 Paris. a vitamine $D$ n'est pas une vitamine dans le vrai sens du mot, elle a en effet une synthèse endogène qui peut remplir totalement les besoins et elle subit des transformations successives afin de devenir active dans les tissus. Les transformations se produisent dans certains tissus; elle est ensuite transportée par le sang et agit dans un autre tissu. Son action cellulaire, actuellement bien mieux connue, récepteur-transcription génétique, fait d'elle une hormone sécostéroïde (9-10 seco-5, 7, I I (I9) cholesta-triene $3 \beta$ ol). On la considère à présent comme une hormone stéroïde calciotrope.

\section{Chimie}

Les vitamines D sont des sécostéroïdes. Leur précurseur, le 7-déhydrocholestérol est lui-même formé à partir du $\delta 7$ cholestérol. Les rayons ultraviolets transforment le 7 déhydrocholestérol par ouverture du cycle $\mathrm{B}$, entre les carbones $\mathrm{C}_{4}-\mathrm{C}_{1}$, en pré-vitamine $\mathrm{D}$; par équilibre isométrique thermique, celle-ci se transforme ensuite en vitamine D. La longueur d'onde des rayons ultra-violets a une grande importance; leur bande active est étroite (290-3I $0 \mathrm{~nm})[\mathrm{I}]$. La source de ces rayonnements, en situation physiologique, est presque uniquement solaire; cependant certaines lampes (fluorescentes) en pro- duisent aussi des quantités non négligeables. La source solaire n'est pas efficace dans n'importe quelle circonstance : pour que les UV arrivent à la surface de la terre il faut que l'angle entre le soleil et l'horizon soit supérieur à $30^{\circ}$; par ailleurs et surtout il ne faut pas qu'ils soient arrêtés par des filtres naturels ou artificiels (nuages, pollution atmosphérique, verres).

\section{Synthèse}

Le lieu de synthèse dépend de l'espèce animale; chez l'homme la synthèse se produit dans l'épiderme en particulier dans la couche basale et muqueuse; le type de peau joue un rôle sur cette synthèse : en effet les peaux colorées ont une capacité de synthèse réduite; cela semble aussi être le cas des peaux riches en kératine. La peau blanche possède une capacité de synthèse très élevée $\left(6 \mathrm{UI} / \mathrm{cm}^{2} / \mathrm{h}\right)$ et une insolation de ro minutes de la surface faciale et des mains est suffisante pour produire un taux vitaminique normal.

\section{Origine exogène}

En l'absence d'exposition solaire, la vitamine $\mathrm{D}$ alimentaire peut remplacer le manque de synthèse. L'absence d'UV, par exemple dans les plongées sous-marines prolongées, fait diminuer de moitié en 3 mois le taux vitaminique circulant, même si l'apport dans le 
régime a été normal. De même dans l'hiver polaire, ce taux vitaminique diminue considérablement si le régime n'est pas très riche en vitamine $D$. L'absorption vitaminique peut avoir une importance dans les maladies digestives, en particulier, celles qui produisent une atrophie de l'entérocyte (maladie cœliaque) ou une déconjugaison des sels biliaires (pullulation microbienne de l'anse borgne), Dans ces cas, l'absorption vitaminique est très diminuée, voire nulle, et les carences vitaminiques avec expressions cliniques (ostéomalacie) fréquentes $[2,3]$.

Cependant, aux USA on a mesuré séparément les taux circulants des dérivés vitamine $D_{2}$ exogènes (car c'est avec cette forme de vitamine $D$ que les aliments sont enrichis), et de la vitamine $\mathrm{D}_{3}$ de production endogène, et on a vu que les dérivés $D_{2}$ ne représentaient que 20 à $30 \%$ de la vitamine circulante; ces résultats ont permis de conclure que lors d'un ensoleillement correct, l'apport vitaminique exogène ne joue qu'un rôle secondaire.

\section{Hydroxylation en $C_{25}$}

La première transformation que subit la vitamine $D$ est une hydroxylation en position $C_{25}$ dans le foie : I heure après une injection de vitamine $\mathrm{D}$ chez le rat carencé, un peu moins de $50 \%$ de celle-ci est trouvée dans le foie. Le 25 hydroxyvitamine $\mathrm{D}(25 \mathrm{OH}$ ) ainsi formé est libéré rapidement dans le sang. Il représente le métabolite vitaminique circulant; chez l'homme en bon équilibre vitaminique, le rapport entre vitamine $\mathrm{D}$ et $25 \mathrm{OHD}$ est de $\mathrm{I} / 5$ à $\mathrm{I} / 4$. Le taux de 25 OHD est un bon reflet du pool vitaminique organique. Actuellement, les dosages de $25 \mathrm{OHD}$ circulant sont fiables et reproductibles; celui qui est le plus employé est basé sur la radiocompétition grâce à une protéine porteuse sérique ou cytosolique rénale. Une des premières constatations faites grâce à ce dosage est l'importance de la variation saisonnière, en fonction des régions, ce qui confirme l'importance de l'ensoleillement sur le taux vitaminique.

Le foie hydroxyle la vitamine D $m / s n^{\circ} 7$ novembre 85 in vivo et in vitro $\left(\mathrm{D}_{2}\right.$ ou $\left.\mathrm{D}_{3}\right)$, cette hydroxylation étant faite aussi sur les dérivés synthétiques comme le tachystérol et le I $\alpha$ hydroxycholécalciférol. Le contrôle de cette hydroxylation est différent selon le substrat : pour les produits synthétiques, l'hydroxylation est rapide et proportionnelle à la quantité de substrat (jusqu'à $25 \mu \mathrm{g}$ de tachystérol ont été testés chez le rat); pour les produits naturels, la linéarité est respectée jusqu'à I $\mu \mathrm{g} / 6 \mathrm{~g}$ de foie [4]; au-delà de ce seuil, et bien que la formation se poursuive, la pente est beaucoup plus faible. Les mécanismes de ce contrôle ne sont pas connus : il n'est pas dépendant de la quantité du $25 \mathrm{OHD}$ car une augmentation artificielle de celui-ci ne diminue pas l'hydroxylation. Les modifications de la chaîne latérale vitaminique inhibent la 25 hydroxylation (25 aza vit D, 25 fluoro vit D) [5]. Dans certaines situations pathologiques ou expérimentales, on a trouvé une modification de cette hydroxylation, en particulier dans les cas où la fonction hépatobiliaire est altérée ou quand une induction enzymatique hépatique (anticonvulsivants; diabète expérimental du rat produit par la streptozocine) est produite.

La localisation subcellulaire de la 25 hydroxylase est encore controversée. Il est surprenant qu'elle soit trouvée préférentiellement dans la fraction microsomale chez les animaux en carence et mitochondriale chez le rat replété en vitamine $D$. De plus, l'hydroxylase microsomale a besoin in vitro d'une fraction cytosolique supplémentaire pour que son activité se manifeste. L'enzyme est une mono-oxygénase, cytochrome $\mathrm{P}_{450}$ dépendante, elle est inhibée par l'oxyde de carbone ainsi que par la métapyrone et nécessite la présence de NADPH.

\section{L'hydroxylation en $C_{1}$ ou en $C_{24}$}

Après une hydroxylation obligatoire en $\mathrm{C}_{25}$, le dérivé se fixe dans le rein pour subir une deuxième hydroxylation sur le carbone i ou le carbone 24. La localisation de ces hydroxylases dans les tubes rénaux a été trouvée très tôt : chez le poulet et le rat, elles se trouvent dans les tubes contournés proximaux [6]. Elles sont mitochondriales; ce sont des monooxydases qui utilisent l'oxygène moléculaire [7]. Celle du I, $25(\mathrm{OH})_{2} \mathrm{D}$ (calcitriol), qui a été la mieux étudiée, exige la présence de magnésium, de NADPH, d'une hémoprotéine, du cytochrome $\mathrm{P}_{45}$, de ferredoxine et d'une ferredoxine reductase [8]. Celle du 24, 25 $(\mathrm{OH})_{2} \mathrm{D}$ n'est pas aussi bien connue; on discute même la participation du cytochrome $\mathrm{P}_{45}$, puisque l'oxyde de carbone ne diminue pas sa formation [9].

La formation des dérivés dihydroxylés est soumise à un contrôle très étroit : de multiples facteurs de la régulation du métabolisme phosphocalcique participent au rétrocontrôle de cette production. In vivo la production du I, 25 $(\mathrm{OH})_{2} \mathrm{D}$ augmente à chaque fois qu'un apport calcique supplémentaire est nécessaire, comme pendant la croissance, la gestation et la lactation. Grâce à des études in vitro, on a pu différencier des facteurs qui agissent directement sur cette hydroxylation, soit pour l'activer, soit pour l'inhiber. Parmi les activateurs, l'action de l'hormone parathyroïdienne (PTH) est une de celles qui a été le plus rapidement individualisée. Les variations ioniques du calcium ou du phosphore agissent très vraisemblablement par son intermédiaire [ro]. Comme l'augmentation de l'adényl cyclase stimule in vitro la I $\alpha$ hydroxylase, il est possible que l'action de la PTH se fasse par son intermédiaire [ II $]$. L'action stimulatrice de la calcitonine in vivo a été expliquée par une action indirecte qui passerait par la PTH; cependant certains travaux réalisés in vitro laisseraient supposer une action directe de cette hormone. D'autres hormones polypeptidiques comme l'insuline, l'hormone de croissance et la prolactine, augmentent in vivo le taux circulant du $1,25(\mathrm{OH})_{2} \mathrm{D}$; toutefois, leur action directe n'est pas encore absolument reconnue.

Des facteurs ioniques autres que le calcium ou le phosphore ont été impliqués; en général, ce sont des facteurs exogènes qui pourraient intervenir lors d'administrations thérapeutiques ou d'intoxications. Par exemple le strontium, les 


\section{RÉFÉRENCES}

5. Onisko BL, Schnocs HK, Deluca HF, Glover RS. Mctabolism and biological activity of 25fluorocholecalciferol, 24-dehydrocholecalciferol and 25-dehydrocholecalciferol in the rat. Biochem 7 1979; 182 : I-9.

6. Brunette MG, Chan M, Ferriere C, Roberts KD. Site of $1.25(\mathrm{OH})_{2}$ vitamine $\mathrm{D}_{3}$ synthesis in the kidney. Nature 1978; 276: 287-9.

7. Kawashima H, Torikai S, Kuburawa K. Localisation of 25-hydroxyvitamin $\mathrm{D}_{3}$ I $\alpha$ hydroxylase and 24 hydroxylase along the rat nephron. Proc Natl Acad Sci USA 1981; 78 : 1 199-2003.

8. Ghazarian JG, Schnoes HK, Deluca HF. Mechanism of 25-hydroxycholecalciferol i $\propto$ hydroxylation. Incorporation of oxygen-18 into the $1 \propto$ position of 25-hydroxycholecalciferol. Biochemistry 1973; 12 : 2555-8.

9. Knutson JC, Deluca HF. 25-hydroxyvitamin $\mathrm{D}_{3}-24$ hydroxylase. Subcellular location and properties. Biochemistry 1974; 13: 1 543-8.

10. Boyle IT, Gray RW, Deluca HF. Regulation by calcium of in vivo synthesis of 1.25 dihydroxycholecalciferol and 21,25 dihydroxycholecalciferol. Proc Natl Acad Sci USA 1971; $68: 2131-4$.

I I. Horiuchi N, Suda T, Takahashi H, Shimazawa $\mathrm{E}$, Ogata $\mathrm{E}$. In vivo evidence for the intermediary role of $3^{\prime} 5^{\prime}$-cyclic AMP in parathyroid hormone induced stimulation of $1 \propto 25$ dihydroxyvitamin $\mathrm{D}_{3}$ synthesis in rats. Endocrino$\log y$ 1977; IOI : 969-74

12. Lambert PW, Stern P, Avioli RC et al. Evidence for extrarenal production of 1.25 dihydroxyvitamin D in man. 7 Clin Invest 1982, $69: 722-5$

13. Barbour GL, Coburn JW, Slatopolski E, Norman AW, Horst RL. Hypercalcemia in an anephric patient with sarcoidosis, evidence for extrarenal generation of 1.25 dihydroxyvitamin D. $N$ Engl 7 Med 1981; 305 : 440-3.

14. Garabedian $M$, Lieberherr $M$, Nguyen TM, Corvol MT, Bailly Dubois M, Balsan S. The in vivo production and activity of 24,25 dihydroxycholecalciferol in cartilage and calvarium. Clin Orthop 1978; 135: 241-8.

15. Nko M, Gruson M, Gueris J et al. Effects of vitamin $\mathrm{D}_{3}$ dihydroxylated metabolites on parathyroid hormone in the rat. Miner Electrolyte biphosphonates, l'éthanol, l'acidose métabolique ou l'acide maléique sont capables de produire une inhibition de l'i $\alpha$ hydroxylase. Il a été prouvé, lors de la mise en évidence des deux hydroxylations rénales $\left(\mathrm{C}_{\mathrm{I}}\right.$ ou C24), qu'elles agissaient en miroir : quand l'activité de l'une augmente, celle de l'autre diminue [9]; ceci est en général respecté, surtout dans les études in vitro. Les éléments que nous venons de voir agissent en sens contraire : les stimulateurs de I $\alpha$ hydroxylase sont des inhibiteurs de la $24 \mathrm{R}$ hydroxylase et inversement.

Bien que le rein soit le producteur le plus important des métabolites dihydroxylés, ils peuvent être produits ailleurs. En dehors du rein, le placenta est capable de synthétiser le I , $25(\mathrm{OH})_{2} \mathrm{D}$. Dans quelques circonstances pathologiques (sarcoïdose) il a été trouvé des taux considérables de $1,25(\mathrm{OH})_{2} \mathrm{D}$ circulant, même chez l'individu anéphrique [12, 13]; par la suite on a démontré que les macrophages pulmonaires des sarcoïdosiques étaient capables de synthétiser le $\mathrm{I}, 25(\mathrm{OH})_{2} \mathrm{D}$ in vitro. Dans l'os, on a trouvé une activité I $\alpha$ hydroxylasique. Cependant, dans les conditions physiologiques, en dehors de la gestation, le principal producteur de $\mathrm{I}_{25}(\mathrm{OH})_{2} \mathrm{D}$ est le tissu rénal. Pour la $24 \mathrm{R}$ hydroxylase, la situation est moins tranchée : même dans le cas d'une anéphrie le $24 \mathrm{R} 25\left(\mathrm{OH}_{2} \mathrm{D}\right.$ est toujours décelable dans la circulation. Il semble que cette hydroxylase soit plus dispersée dans l'organisme : elle a été trouvée dans l'os, le cartilage et l'intestin [ I4].

\section{Parathormone et méta- bolites vitaminiques}

Nous avons déjà vu que la PTH était capable de stimuler l's $\alpha$ hydroxylase et d'inhiber la $24 \mathrm{R}$ hydroxylase. Le $\mathrm{I}, 25(\mathrm{OH})_{2} \mathrm{D}$, par toutes ses actions physiologiques, augmente la concentration du calcium plasmatique et, par son intermédiaire, freine la sécrétion de PTH. Lors de l'administration de calcitriol, en état de carence vitaminique $\mathrm{D}$, le taux de PTH diminue avant que la calcémie n'augmente; cela peut être évidemment en rapport avec le taux de calcium intracellulaire, qui peut se modifier avant qu'il y ait une modification évidente de la calcémie; cependant, in vitro, la sécrétion des cellules parathyroïdiennes est diminuée quand on ajoute dans le milieu du $\mathrm{I}_{2}, 25(\mathrm{OH})_{2} \mathrm{D}$ à une concentration de ${ }_{10}{ }^{-11} M$ [15], ceci laissant penser qu'il a une action directe sur cette sécrétion. Le $24,25(\mathrm{OH})_{2} \mathrm{D}$ diminue aussi la sécrétion parathyroidienne, mais a une concentration I 00 fois supérieure à celle du $\mathrm{I}, 25(\mathrm{OH})_{2} \mathrm{D}_{3}$. On peut donc conclure que la PTH stimule la formation de $\mathrm{I}, 25(\mathrm{OH})_{2} \mathrm{D}_{3}$ et que le $\mathrm{I}, 25(\mathrm{OH})_{2} \mathrm{D}$, en rétrocontrôle, diminue la sécrétion de PTH.

\section{Protéines porteuses vitaminiques}

Les dérivés vitaminiques $\mathrm{D}$ sont liposolubles : leur transport, immédiatement après l'absorption intestinale, se fait comme celui des graisses et des lipoprotéines par voie lymphatique. Rapidement, la vitamine $D$ se lie à une $\alpha_{2}$-globuline plasmatique spécifique du transport vitaminique, la DBP ou vitamin $D$ binding-protein [ $\mathrm{I} 6]$. La protéine porteuse de la vitamine $D$ fait partie des protéines hormonales qui ne traversent pas la membrane cellulaire (seul le "ligand " libre entre dans la cellule pour être fixé à des récepteurs intracellulaires spécifiques), à l'opposé des protéines porteuses de type nutritionnel où l'haloprotéine (complexe protéine porteuse + ligand) est reconnue par la membrane et internalisée [ 1 6]. Les caractéristiques biochimiques de cette protéine sont bien connues : celle des mammifères a un poids moléculaire de 55000 daltons et une constante de sédimentation de $4 \mathrm{~S}$. Cette protéine n'a qu'un seul site de liaison; son affinité pour les différents métabolites n'est pas identique, l'ordre décroissant pour les principaux métabolites étant :

$25 \mathrm{OHD}>24,25(\mathrm{OH})_{2} \mathrm{D}>$ $25,26(\mathrm{OH})_{2} \mathrm{D}>\quad \mathrm{I}, 25(\mathrm{OH})_{2} \mathrm{D}>$ vitamine $\mathrm{D}>$ pré-vitamine $\mathrm{D}$.

Bien que cette protéine ne représente que $0,5 \%$ de la totalité des protéines circulantes chez l'individu normal, on la trouve pourtant dans 
le sérum à une concentration d'environ $300 \mathrm{mg} / \mathrm{l}$, donc en large excès par rapport à la somme des concentrations de tous les métabolites circulants. Son dosage, fait surtout par immunodiffusion, a permis d'observer que sa concentration est relativement stable; des diminutions sensibles ont été observées dans les insuffisances hépatiques et les syndromes néphrotiques et une augmentation lors de la grossesse, probablement par stimulation ostrogénique. En dehors de ces situations particulières il n'a pas été trouvé de perturbation; le pool vitaminique ne modifie pas sa concentration et ceci est compréhensible si l'on sait que $97 \%$ de cette apoprotéine circule sous forme libre. Comme seul le ligand libre est reconnu par la cellule, les fractions vitaminiques libres sont celles qui sont rapidement actives; aussi la DBP représente une forme de stockage de la vitamine.

\section{Actions biologiques}

Du point de vue biologique, le $\mathrm{I}, 25(\mathrm{OH})_{2} \mathrm{D}$ est sans conteste le dérivé le plus actif : il est considéré actuellement comme le produit tissulaire actif[17]. Son action sur l'absorption calcique intestinale est directe : en son absence, le transfert calcique de l'entérocyte est diminué. Son mécanisme d'action est connu : l'entérocyte a des récepteurs chromatiniens spécifiques; après liaison du $\mathrm{I}, 25(\mathrm{OH})_{2} \mathrm{D}_{3}$ à ces récepteurs, il y a transcription, production d'ARN messager et synthèse protéique [ I 8]. Il agit aussi sur les membranes de la cellule et augmente dans celle-ci la perméabilité du calcium, en modifiant les lipoprotéines de la membrane.

D'autre part, le I, $25(\mathrm{OH})_{2} \mathrm{D}$ a des actions sur l'os, mais celles-ci sont synergiques de la PTH. Le I,25 $(\mathrm{OH})_{2} \mathrm{D}$ stimule la résorption osseuse, et, par la libération de calcium qu'elle provoque conjointement avec l'entrée intestinale du calcium, augmente la calcémie [I9]. L'effet du I, $25(\mathrm{OH})_{2}$ D sur les cellules formatrices (ostéoblastes), a été plus difficile à mettre en évidence, car il augmente l'activité des ostéoblastes existant mais non leur différenciation [20] qui semble dé- pendante de l'hormone parathyroïdienne. Concernant la calcification elle même, une controverse demeure : pour les uns il agirait directement [2I], pour d'autres la nouvelle calcification ne serait qu'un effet secondaire de la normalisation des paramètres biologiques ioniques [ 17 ].

L'effet rénal du I, $25(\mathrm{OH})_{2} \mathrm{D}$ est encore plus difficile à préciser : il augmente la réabsorption tubulaire du phosphore, donc agit dans le sens contraire de la PTH; pour le calcium, certaines expériences tendent à montrer qu'il augmente la réabsorption; pour d'autres auteurs, y compris nous-mêmes, en absence de PTH (rats parathyroïdectomisés) l'administration aiguë de I, 25 $(\mathrm{OH})_{2} \mathrm{D}$ augmente considérablement l'élimination urinaire du calcium; ainsi, pour les deux électrolytes, le I,25 $(\mathrm{OH})_{2} \mathrm{D}$ agirait dans le sens contraire de la PTH.

Parmi les autres métabolites, les preuves d'une action physiologique du $24,25(\mathrm{OH})_{2} \mathrm{D}$ sont difficiles à mettre en évidence; en considérant les actions reconnues des modèles expérimentaux, il est sensiblement moins actif que le $\mathrm{I}, 25(\mathrm{OH})_{2} \mathrm{D}$. Pour certains, le $24,25(\mathrm{OH})_{2} \mathrm{D}$ est un produit intermédiaire du catabolisme vitaminique [I 7], puisque l'impossibilité de produire cette hydroxylation dans le cas des dérivés fluorés ne modifie pas l'activité biologique de la molécule. Cependant, bien que l'administration seule du $24,25(\mathrm{OH})_{2} \mathrm{D}$ ne semble agir que faiblement et sur certains paramètres particuliers, en général l'administration simultanée des deux dérivés corrige plus rapidement le défaut de minéralisation qui se produit lors d'une carence[2I]. De plus, le 24, $25(\mathrm{OH})_{2} \mathrm{D}$ retarde l'apparition de l'hypercalcémie produite par le $1,25(\mathrm{OH})_{2} \mathrm{D}$. De là est née une nouvelles hypothèse selon laquelle le $24,25(\mathrm{OH})_{2} \mathrm{D}$ modulerait l'effet du I,25 $(\mathrm{OH})_{2} \mathrm{D}$.

\section{Récepteur du $1,25(\mathrm{OH})_{2} \mathrm{D}$}

Le $\mathrm{I}, 25(\mathrm{OH})_{2} \mathrm{D}$ se comporte du point de vue cellulaire d'une manière analogue à d'autres hormones stéroïdes (œstrogènes, progestérone, androgènes, minéralocorti- coïdes ou glucocorticoïdes) [22-24] Son mécanisme d'action implique successivement : une liaison stéréospécifique avec un récepteur nucléaire chromatinien, la stimulation de la transcription d'ARN messager, et l'induction d'une synthèse protéique "de novo" qui produit une réponse biologique. Le récepteur a été d'abord identifié dans le cytoplasme, puis dans la fraction chromatine[i8]. Le plus largement étudié est celui de l'entérocyte du poulet : il s'agit d'une protéine de 3,5 à $3,7 \mathrm{~S}$, d'un poids moléculaire d'environ 47000 [24] pour les uns, de 80000 pour d'autres. L'activité de liaison est détruite par les dérivés alkylants sulphydriles (N-éthylmaléimide ou iodoacétamide), lorsque le récepteur est libre.

Cependant, les alkylants n'agissent plus après que la liaison s'est produite, ce qui a fait penser qu'un résidu cystéine se trouve à proximité du site de liaison[18]. Les mêmes auteurs considèrent que le phénomène est à entropie positive. L'utilisation de nombreux analogues [18] a montré la spécificité des récepteurs pour le $\mathrm{I}, 25(\mathrm{OH})_{2} \mathrm{D}$.

L'activation du complexe récepteur-stéroïde est faiblement modifiée par la température entre 4 et $23^{\circ} \mathrm{C}$. D'autre part, le coefficient de sédimentation n'est pas modifié par la liaison. Ce sont successivement Haussler [25], puis Norman [18] qui ont purifié le récepteur de 20000 à 40000 fois. La constante de dissociation $(\mathrm{Kd})$ a été trouvée entre 2. $10^{-10} \mathrm{M}\left(4^{\circ} \mathrm{C}\right)$ et $7, \mathrm{I} \cdot 10^{-11} \mathrm{M}$ $\left(25^{\circ} \mathrm{C}\right)$, la constante d'association (Kass) entre $0,9 \cdot 10^{7} \mathrm{M}^{-1} \mathrm{~min}^{-1}$ $\left(0^{\circ} \mathrm{C}\right.$ ) et $9,5 \cdot 10^{8} \mathrm{M}^{-1} \mathrm{~min}^{-1}$ $\left(25^{\circ} \mathrm{C}\right)$, et celle de dissociation (K diss) entre $4,4 \cdot 10^{-5} \mathrm{~min}^{-1}$ $(4 \mathrm{C})$ et $7, \mathrm{I} \cdot 10^{-3} \min ^{-1}\left(25^{\circ} \mathrm{C}\right)$ [18]. Le nombre de sites de liaison dans une cellule serait de 2000 , et la liaison totale de 500 à 700 pmoles de I, $25(\mathrm{OH})_{2} \mathrm{D} / \mathrm{mg}$ de protéine.

Très rapidement, on a mis en évidence ce récepteur spécifique avec les mêmes caractéristiques biochimiques dans les organes cibles vitaminiques : parathyroïde, rein, cellules osseuses. Par la suite, on a trouvé le récepteur dans des cellules qui n'étaient pas considérées comme cibles vitaminiques: pancréas, cancer mammaire, macropha- 


\section{RÉFÉRENCES}

16. Bouillon R, Van Baelen H. Le transport des métabolites de la vitamine D. In: Fournier A, Garabedian M, Sebert JL, Meunier PS, eds. Vitamine $D$ et maladies des os et du métabolisme minéral. Paris: Masson, 1984; 63-9.

17. Deluca HF. Vitamin D metabolism and function. Arch Intern Med 1978; $138: 836-47$.

18. Norman AN, Roth J, Orci L. The vitamin D endocrine system : Steroid Mctabolism, Hormone Receptors, receptors and Biological Response (Calcium Binding proteins). Endocr Rev 1982; 3 : $331-66$.

19. Raisz LG. Direct effects of vitamin D and its metabolites on skeletal tissue. Clin Endocrinol Metab 1980; 9: 27-43.

20. Marie PJ, Travers R. Continuous inf usion of I.25 dihydroxyvitamin $\mathrm{D}$, stimulates bone turnover in the normal young mouse. Calcif Tissue $I n t$ $1983 ; 35^{\text {t }} 4$ 1 8-25.

21. Bordier P, Miravet L, Marie P, et al. Action des métabolites de la vitamine $\mathrm{D}$ sur la minéralisation du tissu osseux et les troubles du métabolisme phosphocalcique au cours de l'ostéomalacic hypovitaminique D. Revue du Rhumatisme 1978; $45: 241-8$.

22. O'Malley BW, Aiger M, Baulicu EE. Acidophilic activation of steroid receptors. Biochemistry 1973; 183: 610-5.

23. Bruchousky $\mathrm{N}$, Wilson JD. The intranuclear binding of testosterone and $5 \alpha$ androstan$17 \beta$ ol-3 one by rat prostate. 7 Biol Chem 1968 ; 243: 5953-60.

24. Wecksler WR, Norman AW. Biochemical properties of I $\propto 25$ hydroxyvitamin $D$ receptors (reviews). I Steroid Biochem 1960; 13 : 977-89.

25. McCain RA, Haussler MR, Okrent D, Hughes MR. Partial purification of the chick intestinal receptor for 1.25 dihydroxyvitamin D by ion-exchange and blue dextran sepharose chromatography. FEBS LETT $1978 ; 86: 65-78$.

26. Manolagas SC, Deftos LJ. The vitamine D endocrine system and the hematolymphopoietic tissue Ann Intern Med 1984; 100 : 144-6.

27. Yetgin S, Ozsoylu S. Myeloid metaplasia in vitamine D deficiency rickets. Scand 7 Haematol 1982; 28 : $180-3$.

28. Suda $T$, Abe E, Miyaura $C$, Tanaka $H$, Shiina Y, Kuribayashi T. Vitamin D in the differentiation of myeloid leukemia cell. In: Kumar $R$ ed. Vitamin D. Boston: Martinus Nijhoff 1984; $343-63$.

29. Bhalla AK, Amento EP, Clemens TL, Holick M, Krane SM. Specific high affinity receptors for I.25 dihydroxyvitamin $D_{3}$ in human peripheral blood mononuclear cells: presence in monocytes and induction in $\mathrm{T}$ lymphocytes following activation. 3 Clin Endocrinol Metab 1983; 57 : $1308-10$.

30. Tsoukas CD, Provvedini DM, Manologas SC. 1.25 dihydroxyvitamin $D_{3}$. A novel immuno-

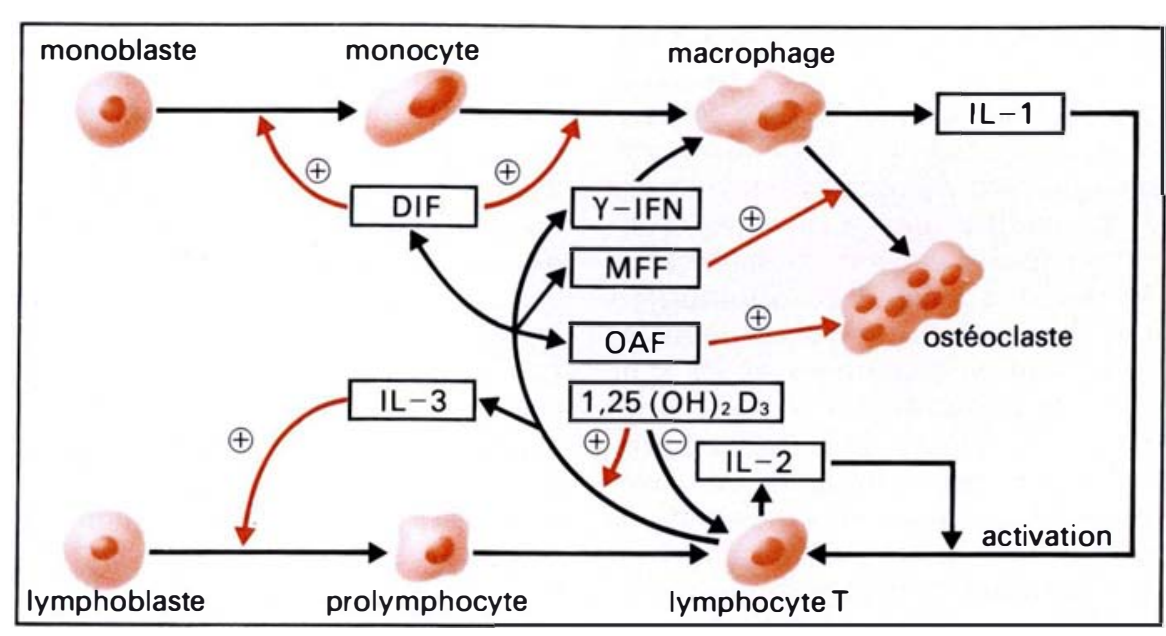

Figure I. Actions immunomodulatrices du 1.25 dihydroxyvitamine D.

$I L$ : interleukine; $M F F$ : macrophage fusion factor; $O A F$ : osteoclast activating factor; $\gamma-I F N$ : $\gamma$ interferon; $D I F$ : differentiating inducing factor.



Figure 2. Actions cellulaires du 1.25 dihydroxyvitamine D.

$I L$ : interleukine; $B G P$ : bone gla protein.

ges, monocytes, lymphocytes T-B malins ou activés, thymoblastes.

Ces acquisitions toutes récentes élargissent le champ d'action de la vitamine D.

\section{Actions immunomodulatrices}

La production des interleukines est modifiée par le calcitriol : il stimule la sécrétion d'interleukine I d'origine macrophagique et lymphocytaire [26]. Cette interleukine inhibe la synthèse de l'ostéocalcine ou gla-protéine. Cette protéine est une calcium binding-protein de petit poids moléculaire $(6000)$, synthétisée par l'ostéoblaste, qui se lie à l'hydroxylapatite et s'accumule ainsi dans l'os, ce qui suggère que cette protéine joue un rôle dans les processus de minéralisation osseuse. L'interleukine I pourrait donc constituer le signal d'une rétrorégulation négative du taux de sécrétion de cette protéine qui est stimulée par le $1,25(\mathrm{OH})_{2} \mathrm{D}_{3}$. En outre, l'interleukine I contribue à l'action inhibitrice du $\mathrm{I}_{25}(\mathrm{OH})_{2} \mathrm{D}_{3}$ sur la synthèse de collagène par l'ostéoblaste. Par ailleurs, cette interleukine stimule la résorption osseuse et participe avec l'interleukine 2 à 
l'activation du lymphocyte $T$. Le I, $25(\mathrm{OH})_{2} \mathrm{D}_{3}$ inhibe également la production lymphocytaire de cette interleukine 2 [26].

D'autre part, le I, $25(\mathrm{OH})_{2} \mathrm{D}_{3}$ stimule la sécrétion d'autres lymphokines qui activent la différenciation cellulaire de cellules souches médullaires (interleukine 3 ) et des cellules de la lignée monocyte-macrophage vers l'ostéoclaste (differentiating inducing factor, macrophage fusion factor, osteoclast activating factor). Il stimule également la production lymphocytaire d'interféron $\gamma$ qui à son tour stimule la i $\alpha$ hydroxylase macrophagique (figure I).

\section{Actions cellulaires}

La localisation cellulaire des récepteurs au I,25 $(\mathrm{OH})_{2} \mathrm{D}$ dans les cellules hématopoiétiques a permis de penser que le calcitriol pouvait être impliqué dans la régulation et/ou l'activité cellulaire [26] (figure 2). Déjà en 1956, il avait été observé qu'un régime pauvre en vitamine $D$ augmentait de plusieurs centaines de fois le nombre des mastocytes de la moelle osseuse. Plus récemment, il a été signalé chez l'enfant rachitique une anémie avec hématopoièse extramédullaire corrigée par l'administration vitaminique [27]. D'autre part, la carence vitaminique est associée à une plus grande susceptibilité infectieuse. De plus, la réponse à un stimulus inflammatoire non-spécifique serait diminuée dans ces cas et les leucocytes périphériques auraient une diminution de leur mobilité et de leur capacité de phagocytose.

Du point de vue expérimental, les macrophages et leucocytes de la souris carencée en vitamine $D$ ont une diminution de leur migration spontanée et de leur capacité phagocytaire in vitro, qui est corrigée par l'adjonction du calcitriol au milieu de culture. L'action cellulaire du $\mathrm{I}, 25(\mathrm{OH})_{2} \mathrm{D}$ a acquis une nouvelle dimension lorsqu'on observa qu'il était capable d'induire in vitro la différenciation des cellules myéloïdes leucémiques, murines ou humaines, en macrophages [28].

Une implication thérapeutique pourrait même en être déduite, en considérant que l'administration de I,25 $(\mathrm{OH})_{2} \mathrm{D}$ prolonge nettement la survie des souris inoculées avec des cellules leucémiques.

Dans les monocytes circulants, des récepteurs en tout point identiques à ceux des organes cibles ont été trouvés [29]. La lignée monocytesmacrophages est capable de produire une résorption osseuse in vitro. L'ostéoclaste (macrophage osseux) dérive aussi des monocytes; cependant, dans l'ostéoclaste différencié, il n'a pas été encore mis en évidence de récepteurs au calcitriol, alors qu'on les a trouvés dans les ostéoblastes (cellules formatrices de l'os). Les monocytes contrôlent en outre la production d'une lymphokine OAF (osteoclast activating factor) sécrétée par les lymphocytes stimulés par la phytohémagglutinine [30]. L'administration de phytohémagglutinine chez l'animal accélère la différenciation des ostéoclastes et augmente leur nombre.

Les lymphocytes, par contre, ne démasquent leurs récepteurs au calcitriol que lorsqu'ils sont stimulés, ou malins. Les lymphocytes $T$ et $B$ normaux n'ont pas de récepteurs. Quant aux cellules thymiques, les thymoblastes ont des récepteurs mais non les thymocytes. Cela laisse supposer une association entre les récepteurs du calcitriol et l'activité mitotique cellulaire. Sous l'action du $1,25(\mathrm{OH})_{2} D_{3}$, on a également décrit une augmentation du temps de mitose de cellules de mélanome malin, une inhibition de la prolifération des cellules de cancer mammaire humain, avec présence de récepteurs au $1,25(\mathrm{OH})_{2} \mathrm{D}_{3}$ dans ce tissu tumoral; une inhibition de la croissance et une stimulation de la différenciation de cellules de sarcome ostéogénique (dotées de propriétés ostéoblastiques) liées à la présence d'un récepteur au I, $25(\mathrm{OH})_{2} \mathrm{D}_{3}$. Tous ces phénomènes permettent de penser que le calcitriol intervient lors de la différenciation cellulaire et de la réponse immunitaire. En conclusion, la vitamine D ne doit pas être aujourd'hui considérée seulement comme une hormone stéroïde calciotrope, car elle est douée d'actions beaucoup plus complexes et générales que celles considérées jusqu'à présent. Notamment, une action sur les fonctions immunitaires et la différenciation cellulaire

\section{Summary}

Beside its classical actions on intestine (stimulation of calcium and phosphate absorption), kidney (stimulation of calcium tubular reabsorption) and bone (calcium mobilization), the I.25 dihydroxycholecalciferol

$\left(\mathrm{I} .25(\mathrm{OH})_{2} \mathrm{D}_{3}\right)$, the most active metabolite of vitamin $\mathrm{D}$, has been recently found to act as an immunomodulator and a cellular differentiating agent. All these actions are mostly mediated through a receptor-mediated mechanism, similar to that of other steroid hormones. The main gene products, presently identified, include the family of calcium binding proteins which regulate probably the intracellular calcium transport. Numerous tissues and cells process I. $25(\mathrm{OH})_{2} \mathrm{D}_{3}$ receptors illustrating the ubiquitous and general effects of $1.25(\mathrm{OH})_{2} \mathrm{D}_{3}$ so far underestimated.

\section{TIRÉS A PART}

L. Miravet : Inserm U 18, hôpital Lariboisière, 6, rue Guy-Patin, 75010 Paris. 